\title{
Comportamento Funcional dos Portadores de Marcapasso Convencional Submetidos a Ressincronização Cardíaca
}

Functional behavior of patients with conventional pacemakers undergoing cardiac resynchronization

\author{
Rodrigo Tavares Silva, Martino Martinelli Filho, Carlos Eduardo Batista de Lima, Daniela Garcia Moreno Cabral Martins, \\ Silvana Angelina Dorio Nishióka, Anísio Alexandre Pedrosa, Elizabeth Sartori Crevelari, Roberto Costa, Eduardo \\ Argentino Sosa, José Antonio Franchini Ramires \\ Instituto do Coração do Hospital das Clínicas da Faculdade de Medicina da Universidade de São Paulo, São Paulo, SP - Brasil
}

\section{Resumo}

Fundamento: A terapia de ressincronização cardíaca (TRC) é eficiente no tratamento de pacientes com insuficiência cardíaca (IC), disfunção ventricular grave e bloqueio intraventricular. O marcapasso convencional (MPC) em região apical de ventrículo direito provoca alterações da seqüência de ativação normal do coração semelhante às do BRE. Nesse sentido, pacientes com MPC e IC avançada poderiam ser candidatos a TRC, mas reduzidas casuísticas foram avaliadas e não há conclusões definitivas.

Objetivo: Analisar o comportamento clínico-funcional da terapia de ressincronização cardíaca (TRC) nos portadores de marcapasso convencional.

Métodos: Pacientes com MPC, IC-CF(NYHA) III/IV refratária a terapêutica medicamentosa, fração de ejeção do ventrículo esquerdo (FEVE) $<35 \%$, foram submetidos a TRC. O comportamento clínico-funcional foi avaliado prospectivamente após seis meses. A redução de uma CF-IC foi estabelecida como resposta efetiva ao procedimento. Foram analisados: duração do QRS (ECG), diâmetro diastólico (DDVE), diâmetro sistólico do ventrículo esquerdo (DSVE) e FEVE ao ECO. A análise estatística utilizou os testes t de Student pareado e a correlação de Spearman.

Resultados: Vinte e nove pacientes com idade média de 61,5 anos foram estudados. Seis eram do sexo feminino e houve predomínio da cardiomiopatia chagásica. Em seguimento clínico de $22,7 \pm 13$ meses, $86,2 \%$ dos pacientes melhoraram com a TRC. Nesse grupo, a FEVE média aumentou em 18\% $(p=0,013)$; houve redução da duração do QRS em $11,8 \%$ $(p=0,002)$ e não houve redução significativa dos diâmetros intracavitários do ventrículo esquerdo.

Conclusão: A TRC é efetiva para pacientes com MPC e IC avançada porque proporciona taxa elevada de responsivos (86,2\%), melhora significativa da FEVE e redução da duração do QRS. (Arq Bras Cardiol 2008; 90(2): 151-156)

Palavras-chave: Marcapasso cardíaco artificial, bloqueio de ramo, insuficiência cardíaca congestiva, estimulação cardíaca artificial.

\section{Summary}

Background: Cardiac resynchronization therapy (CRT) is an efficient treatment for patients with heart failure (HF), severe ventricular dysfunction and intraventricular block. Conventional pacemakers (CPM) implanted in the right ventricular apical area cause alterations in the normal sequence of cardiac activation similar to those induced by LBBB (left bundle-branch block). Therefore, patients with CPM and advanced HF could be candidates to undergo CRT, but as only small numbers of patients have been evaluated so far, definitive conclusions are lacking.

Objective: To assess the clinical and functional outcome of cardiac resynchronization therapy (CRT) in patients with conventional pacemakers.

Methods: Patients with CPM, who were in NYHA HF functional class III/IV class refractory to drug therapy, and left ventricular ejection fraction $($ LVEF) $)<35 \%$ underwent CRT. Patients' clinical-functional behavior was assessed prospectively six months after the procedure. The improvement of one HF-functional class was set as an effective response to the procedure. The following was assessed: QRS duration (ECG), diastolic diameter (LVDd), left ventricular systolic diameter (LVSd) and LVEF seen on the echocardiogram. For the statistical analysis, Student's paired t test and Spearman's correlation were used.

Results: Twenty-nine patients (mean age 61.5) were evaluated. Of these, six were females, and chagasic cardiomyopathy was predominant. During the clinical follow-up of $22.7 \pm 13$ months, $86.2 \%$ of the patients benefited from CRT. Within this group, the mean LVEF increased by $18 \%$ $(p=0.013), Q R S$ duration dropped by $11.8 \%(p=0.002)$ and no significant reduction in left ventricular intracavitary diameters was observed.

Conclusion: CRT is effective for patients with CPM and advanced HF as it yields a high rate of response (86.2\%), significantly improves LVEF and reduces QRS duration. (Arq Bras Cardiol 2008;90(2):138-143)

Key words: Pacemaker, artificial; bundle-branch block; heart failure, congestive; cardiac pacing, artificial.

Full texts in English - http://www.arquivosonline.com.br

Correspondência: Rodrigo Tavares Silva •

Rua João Moura, 870 - 05412002 - Pinheiros, São Paulo, SP - Brasil

E-mail: rotasil@ig.com.br

Artigo recebido em 25/03/07; revisado recebido em 05/06/07; aceito em 03/10/07. 


\section{Artigo Original}

\section{Introdução}

Distúrbios de condução intraventricular são manifestações freqüentes em pacientes com insuficiência cardíaca (IC) ${ }^{1}$. De modo geral, associam-se à gravidade do acometimento miocárdico e, de modo independente, com maiores taxas de morbidade e mortalidade nessa população ${ }^{2,3}$. Aproximadamente 25\% a 50\% dos pacientes com IC avançada apresentam complexo QRS com duração prolongada, e a maioria tem morfologia de bloqueio de ramo esquerdo $(B R E)^{1,3}$. Sabe-se que esse, por sua vez, determina alterações da seqüência de ativação do ventrículo esquerdo (VE) ocasionando atraso da contração da parede lateral em relação ao septo interventricular ${ }^{3}$.

A estimulação cardíaca artificial apical do ventrículo do direito (VD), provocada pelo marcapasso convencional (MPC), promove seqüência de ativação ventricular similar à que ocorre no BRE espontâneo ${ }^{4-6}$. Dependendo do grau de acometimento miocárdico, essa dissincronia elétrica induzida pode ser responsável por dissincronia ventricular mecânica e agravar a evolução da cardiopatia de base. Isso porque, na presença de disfunção sistólica importante, ocorre aceleração do dano celular miocárdico, alterações da geometria ventricular, crescimento atrial, piora da regurgitação mitral e conseqüente deterioração hemodinâmica ${ }^{6}$. Recentemente, evidências clínicas vêm surgindo com a publicação dos ensaios DAVID study ${ }^{7}$, MADIT- $I{ }^{8}$, Mode Selection Trial ${ }^{9}$ e PAVE Trial ${ }^{10}$. Sub-análises desses estudos relacionaram a estimulação cardíaca artificial com maiores taxas de internações hospitalares por IC, maior ocorrência de fibrilação atrial e insuficiência mitral.

Nos últimos anos, a terapia de ressincronização cardíaca (TRC) tem demonstrado eficiência no manuseio de pacientes com IC sistólica avançada, disfunção ventricular grave e bloqueio intraventricular (QRS >120 ms). Entretanto, essas evidências científicas estão baseadas em estudos de larga escala que invariavelmente excluíram pacientes com MPC ${ }^{11}$ ${ }^{14}$. Höijer e cols. ${ }^{15}$ demonstraram em 10 pacientes com IC e MPC que o implante do sistema biventricular proporciona efeitos benéficos em parâmetros clínicos, funcionais e neuroendócrinos. Outros estudos com casuísticas limitadas também evidenciaram resultados semelhantes ${ }^{1,4}$.

O presente estudo tem por objetivo avaliar o comportamento clínico-funcional de pacientes com IC e MPC em região apical do VD submetidos à TRC.

\section{Método}

\section{Casuística}

Os pacientes do estudo foram selecionados dentre os 484 portadores de ressincronizador cardíaco (RC), da coorte acompanhada prospectivamente na Unidade de Estimulação Cardíaca Artificial do Instituto do Coração - HCFMUSP, até dezembro de 2004.

Os critérios de inclusão foram baseados nas características clínico-funcionais dos pacientes pré-implante do RC: 1) BRE induzido por MPC; 2) cardiomiopatia dilatada classe funcional III/IV de IC pela New York Heart Association (IC-NYHA); 3) fração de ejeção do ventrículo esquerdo (FEVE) $\leq 35 \%$; e 4) refratariedade ao tratamento clínico otimizado, constituído por, no mínimo, inibidor da enzima conversora de angiotensina ou bloqueador dos receptores de angiotensina, betabloqueador e espironolactona; todos em doses máximas toleráveis, a menos que houvesse alguma contra-indicação. Além desses, fazia parte do arsenal terapêutico cardiovascular a utilização de diuréticos, vasodilatores, digital, bloqueadores dos canais de cálcio e antiarrítmicos entre outros, de acordo com a necessidade individual e tolerância de cada paciente.

Não foram incluídos pacientes portadores de cardiodesfibrilador implantável, portadores de marcapasso com disfunção do sistema implantado, pós-transplante cardíaco, doença terminal com expectativa de sobrevida inferior a um ano, eventos cardiovasculares recentes $<3$ meses, doença pulmonar obstrutiva grave e pacientes com seguimento em outras instituições.

O critério adotado para avaliação de resposta clínica à TRC, em seguimento clínico mínimo de seis meses, foi a redução de pelo menos uma classe funcional de IC-NYHA. Foram analisados ainda: 1) variação da duração do complexo QRS; 2) variação dos diâmetros diastólico e sistólico do VE (DDVE e DSVE); e 3) comportamento da FEVE.

As avaliações realizadas, previamente ao implante do sistema biventricular, foram: exame físico geral; classe funcional de IC-NYHA; eletrocardiograma de 12 derivações; ecocardiograma transtorácico e flebografia digital de membros superiores. O seguimento clínico dos pacientes foi realizado na clínica de estimulação cardíaca artificial do Instituto do Coração, inicialmente nos $10^{\circ}$ e $30^{\circ}$ dias pós-implante do RC, e a partir de então, a cada trimestre. Nas consultas de retorno, os pacientes realizavam avaliação clínica completa, eletrocardiográfica e eletrônica do sistema de estimulação.

\section{Implante do ressincronizador cardíaco}

Previamente à cirurgia, todos os pacientes foram submetidos a flebografia digital de membros superiores para a avaliação da patência venosa e planejamento da estratégia cirúrgica. Os cabos-eletrodos prévios, quando normofuncionantes, foram mantidos sempre que possível. O cabo-eletrodo adicional para estimulação do VE foi implantado, preferencialmente, por abordagem transvenosa do seio coronário e posicionado em veias que drenam a parede lateral ou póstero-lateral do VE. A estenose de veias subclávias/inominadas relacionadas ao dispositivo previamente implantado não foi critério de exclusão para estudo. Quando isso ocorreu, implantou-se novo sistema de estimulação biventricular no lado contralateral; nos casos em que os geradores já estavam em região torácica esquerda, os pacientes foram submetidos a toracotomia lateral esquerda minimamente invasiva para implante do cabo-eletrodo adicional em ventrículo esquerdo.

Todos os geradores de pulso foram substituídos por dispositivos ressincronizadores disponíveis no mercado no período do estudo. Nos procedimentos iniciais, foram utilizados adaptadores em Y para a conexão entre os caboseletrodos de VD e VE. Posteriormente, passaram a ser utilizados sistemas com canais independentes.

A programação do modo de estimulação biventricular ficou a critério da equipe clínica de estimulação cardíaca artificial. Os intervalos atrioventricular e interventricular 
foram programados de maneira individualizada, dirigida por ecocardiografia.

\section{Ecocardiograma transtorácico}

A avaliação ecocardiográfica utilizou o modo M para análise do DDVE e DSVE em milímetros ( $\mathrm{mm}$ ) e o modo bidimensional para a FEVE em porcentagem (\%). O serviço de Ecocardiografia do Instituto do Coração foi o responsável pela realização dos exames, seguindo as diretrizes estabelecidas pela Sociedade Brasileira de Ecocardiografia, e conforme à solicitação da equipe responsável pelo seguimento ambulatorial dos pacientes. O exame ecocardiográfico após a TRC foi realizado com intervalo mínimo seis meses após o procedimento cirúrgico de implante do sistema biventricular.

\section{Eletrocardiograma de 12 derivações}

A duração do complexo QRS (milissegundos) estimulado foi avaliada por meio do eletrocardiograma de superfície de 12 derivações com calibração de $0,1 \mathrm{mV} / \mathrm{mm}$ e velocidade do papel de $25 \mathrm{~mm} / \mathrm{s}$. Considerou-se a medida eletrônica realizada pelo aparelho ou quando feita manualmente, o menor intervalo de tempo desde a emissão da espícula do marcapasso até o final do complexo QRS em qualquer das derivações.

\section{Análise estatística}

A taxa de resposta efetiva à TRC foi avaliada pelo cálculo da porcentagem de pacientes que apresentaram redução de uma classe funcional de IC-NYHA. Utilizou-se o teste de correlação de Spearman para a análise das variáveis classificatórias e o teste de $t$ Student pareado para análise da variação da duração do complexo QRS e do comportamento dos parâmetros ecocardiográficos pré e pós-TRC. $O$ valor de $P$ foi considerado significante quando inferior a 0,05.

\section{Resultados}

Vinte e nove pacientes com BRE induzido por MPC foram submetidos a otimização para TRC no período de março de 1997 a dezembro de 2004. As características clínico-funcionais da casuística estão incluídas na tabela 1. A idade média do grupo foi de 61,5 anos, e $79 \%$ eram do sexo masculino. Houve predomínio da cardiomiopatia chagásica (52\%) e da idiopática (21\%). Em relação à CF-IC, 86\% do grupo estavam em CF III. A terapia medicamentosa cardiovascular estava otimizada, e mais de $75 \%$ dos pacientes estavam em uso concomitante de IECA/ ARA, betabloqueador e espironolactona. Os valores médios dos parâmetros ecocardiográficos foram: FEVE de 27,7\%; DDVE de 69,7 mm; e DSVE de 63,8 mm. Dezoito pacientes estavam em modo de estimulação sincronizado (DDD) e a duração média do QRS estimulado foi de 185 ms. O tempo decorrido entre o implante do marcapasso inicial e da otimização para TRC foi de 114,3 $\pm 80,3$ meses. O seguimento clínico do grupo pós-procedimento cirúrgico foi $22,7 \pm 13$ meses.

A cateterização do seio coronário para implante do caboeletrodo do VE ocorreu em 26 pacientes (89,6\%); em 25 pacientes $(86,2 \%)$ o sistema foi implantado com sucesso por via transvenosa. Em um paciente, apesar da cateterização do seio coronário, houve escassez de veias tributárias que
Tabela 1 - Características da casuística

\begin{tabular}{|c|c|}
\hline Total de pacientes & 29 \\
\hline Idade em anos (DP) & $61,5 \pm 17$ \\
\hline \multicolumn{2}{|l|}{ Sexo (\%) } \\
\hline Feminino & $6(21 \%)$ \\
\hline Masculino & $23(79 \%)$ \\
\hline \multicolumn{2}{|l|}{ Cardiopatia de base (\%) } \\
\hline Chagásica & $15(52 \%)$ \\
\hline Idiopática & $6(21 \%)$ \\
\hline Isquêmica & $5(17 \%)$ \\
\hline Valvar & $2(7 \%)$ \\
\hline Congênito & $1(3 \%)$ \\
\hline \multicolumn{2}{|c|}{ Indicação primária de MPC (\%) } \\
\hline DNS+BAV $1^{\circ}$ & $2(7 \%)$ \\
\hline BAV $2^{\circ}$ & $1(3 \%)$ \\
\hline $\mathrm{BAV} 3^{\circ}$ & $26(90 \%)$ \\
\hline \multicolumn{2}{|c|}{ Classe funcional de IC (NYHA) (\%) } \\
\hline III & $25(86 \%)$ \\
\hline IV & $4(14 \%)$ \\
\hline \multicolumn{2}{|c|}{ Medicação cardiovascular (\%) } \\
\hline IECA/ARA & $25(86 \%)$ \\
\hline Betabloqueador & $22(76 \%)$ \\
\hline Espironolactona & $22(76 \%)$ \\
\hline Diuréticos & $25(86 \%)$ \\
\hline Digital & $18(62 \%)$ \\
\hline Amiodarona & $12(41 \%)$ \\
\hline \multicolumn{2}{|c|}{ Parâmetros ecocardiográficos (DP) } \\
\hline $\mathrm{DDVE}(\mathrm{mm})$ & $69,7 \pm 10$ \\
\hline DSVE (mm) & $63,8 \pm 7$ \\
\hline FEVE $(\%)$ & $27,7 \pm 4,8$ \\
\hline \multicolumn{2}{|l|}{ Modo de estimulação (\%) } \\
\hline Unicameral (VVI) & $11(38 \%)$ \\
\hline Dupla câmara (DDD) & $18(62 \%)$ \\
\hline \multicolumn{2}{|l|}{ QRS estimulado basal } \\
\hline Duração (ms) & $185 \pm 34$ \\
\hline
\end{tabular}

$D P$ - desvio padrão; DNS - doença do nó sinusal; BAV - bloqueio atrioventricular; IECA/ARA - inibidores da enzima conversora de angiotensina/antagonistas dos receptores de angiotensina; DDVE - diâmetro diastólico do ventrículo esquerdo; DSVE - diâmetro sistólico do ventrículo esquerdo; FEVE - fração de ejeção do ventrículo esquerdo; IC - insuficiência cardíaca; ms: milissegundos.

impossibilitaram fixação do cabo-eletrodo. Esse paciente já apresentava dispositivo prévio implantado em região torácica esquerda, havendo conversão para toracotomia lateral minimamente invasiva. A falha na cateterização endovenosa do seio coronário ocorreu em três pacientes; nesses, o implante do cabo-eletrodo de VE também foi por minitoracotomia lateral esquerda. 
Silva e cols.

Ressincronização Cardíaca em Portadores de Marcapasso Convencional

\section{Artigo Original}

A taxa de estenose de veias subclávias e inominadas que impossibilitou a passagem do cabo eletrodo adicional foi de $6,8 \%$ (dois pacientes); optou-se pelo implante de novo sistema de estimulação no lado contralateral e pelo abandono do sistema antigo. Não houve complicações relacionadas ao procedimento cirúrgico.

No seguimento clínico de 22,7 meses, a taxa de resposta clínica efetiva a TRC foi de $86 \%$ dos pacientes. Ademais, a maioria desses $(82,7 \%)$ permaneceu em CF I/II de IC-NYHA (Figura 1). Não houve modificação significativa na terapia medicamentosa durante o seguimento clínico.

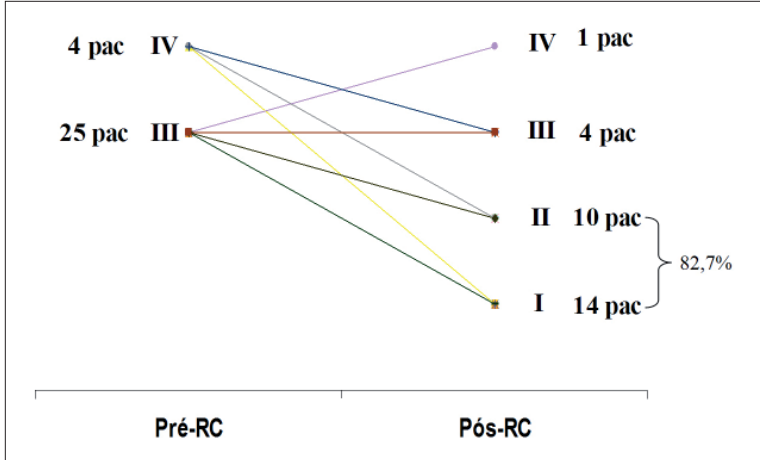

Fig. 1 - Comportamento da classe funcional dos pacientes (IC-NYHA) pré e pós a RC. Após a RC, $82,7 \%$ dos pacientes permaneceram em CF-IC I/II. RC - ressincronização cardíaca; IC - insuficiência cardíaca; CF - classe funcional; NYHA - New York Heart Associattion.

A análise dos parâmetros ecocardiográficos demonstrou melhora de 18\% na FEVE média dos pacientes após a TRC $(P=0,013)$. Não houve redução significante dos diâmetros intracavitários do $\mathrm{VE}$; ocorrendo uma variação de $3,5 \mathrm{~mm}$ no DDVE $(P=0,29)$ e de $4,3 \mathrm{~mm}$ no DSVE $(P=0,15)$ com o implante do RC (Figura 2). O tempo médio decorrido entre a TRC e o exame ecocardiográfico pós-procedimento foi de 10,8 meses.

Ocorreu redução significativa de $11,8 \%$ na duração do complexo QRS após a estimulação biventricular $(P=0,002)$. No entanto, não existiu correlação entre a redução da duração do QRS e melhora clínica ou de parâmetros ecocardiográficos.

\section{Discussão}

Os achados deste estudo indicam que a TRC é eficaz para pacientes com cardiomiopatia dilatada refratária ao tratamento clínico e BRE induzido pelo MPC. Trata-se de uma das maiores casuísticas da literatura a avaliar esse perfil de pacientes, destacando-se pela elevada prevalência da cardiomiopatia chagásica (52\%) e pelo longo tempo de seguimento clínico pós-implante do RC (22,7 meses). Esses achados diferem de estudos similares, em que houve predomínio da cardiomiopatia dilatada idiopática e o seguimento clínico foi bem inferior (6-12 meses) ${ }^{11-13}$.

Nos últimos anos, a TRC vem sendo intensivamente estudada em pacientes com BRE espontâneo e IC avançada ${ }^{11-14}$

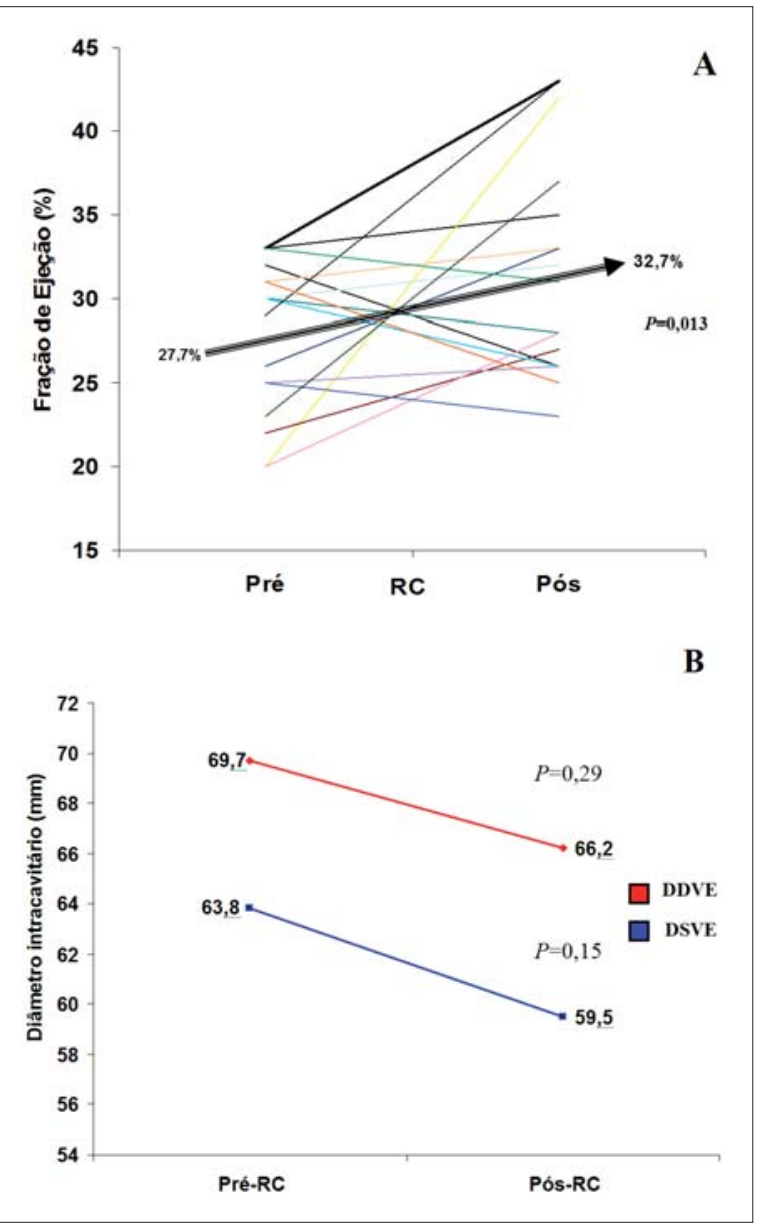

Fig. 2 - Análise dos parâmetros ecocardiográficos pré e pós RC. 0 gráfico A evidencia as variações individuais da FEVE de cada paciente antes e após a RC. Houve melhora de $18 \%$ na FEVE média do grupo após a $R C(P=0,013)$. $O$ gráfico $B$ demonstra a variação (não-significativa) dos diâmetros intracavitários (DDVE/DSVE) pós-RC. FEVE - fração de ejeção do ventrículo esquerdo; $R C$ : ressincronização cardíaca; DDVE - diâmetro diastólico do ventrículo esquerdo; DSVE - diâmetro sistólico do ventrículo esquerdo; IC - insuficiência cardíaca; $\mathrm{mm}$ : milímetros.

No entanto, a dissincronia ventricular induzida pelo MPC em região apical de VD ainda carece de respaldo científico.

O BRE induzido pelo MPC resulta em dissincronia interventricular, promovendo atraso de 30-180 ms na ativação do VE, assim como dissincronia intraventricular conseqüente à alteração da seqüência de ativação mecânica (ápice $\rightarrow$ base em vez de base $\rightarrow$ ápice). Além disso, a estimulação apical do VD promove distribuição heterogênea do estresse miocárdico, adelgaçamento das regiões precocemente ativadas em oposição às tardiamente ativadas que se tornam mais espessas. Essa heterogeneidade regional resulta em remodelamento do $\mathrm{VE}$, que em última análise leva a alterações contráteis e hemodinâmicas, reduzindo sua eficiência funcional. Mecanismos adicionais como alterações neuro-endócrinas, alterações nos canais de cálcio tipo-L, nas proteínas de expressão, desarranjo miofibrilar, fibrose, deposição de tecido gorduroso, defeitos de perfusão tecidual e um variado 
grau de regurgitação mitral contribuem diretamente para o comprometimento da função cardíaca ${ }^{16}$.

Inicialmente, para a correção desses distúrbios foram utilizados alguns fármacos, sobretudo betabloqueadores que, entretanto, não apresentaram resultados sustentados ${ }^{17}$.

A TRC surgiu, em 1996, como uma alternativa terapêutica invasiva, realizada por toracotomia lateral esquerda e por isso inicialmente não provocou muito entusiasmo. A introdução da técnica transvenosa por cateterização do seio coronário e o desenvolvimento tecnológico de cabos-eletrodos de VE tornaram o procedimento menos invasivo, e sua indicação cresceu gradativamente em todo o mundo. Nesta casuística, houve baixa taxa de estenose grave do sistema venoso $(6,8 \%)$ relacionado ao dispositivo implantado previamente, permitindo elevado índice de sucesso mediante a técnica transvenosa por cateterização do seio coronário (86,2\%). Atualmente, no Instituto do Coração - FMUSP são realizados aproximadamente 95 implantes por ano de ressincronizadores cardíacos associados ou não a cardiodesfibriladores, dentre os quais $85 \%$ são realizados por via transvenosa através do seio coronário.

Os ensaios clássicos que consagraram a TRC como terapêutica coadjuvante para pacientes com IC grave e distúrbios de condução intraventriculares demonstraram invariavelmente melhora clínico-funcional11-14. Em nosso estudo, a taxa de resposta clínica a TRC foi de $86,2 \%$, valor superior aos achados de Reuter e cols. $(82,4 \%)^{18}$ e de Molhoek e cols. $(68 \%)^{19}$, ressaltando-se a utilização de critérios semelhantes. Houve redução $>1$ (uma) CF-IC em 55,1\% dos nossos pacientes, e após a TRC 82,7\% permaneceram em classe funcional I/II (IC-NYHA). Apesar da subjetividade aparente do método utilizado para avaliação da resposta clínica, a sua validação está demonstrada nesse perfil de pacientes com sintomas limitantes ${ }^{20-22}$. Outras medidas de avaliação clínica após a TRC, como questionário de qualidade de vida, teste de caminhada de seis minutos ou teste ergoespirométrico, não foram realizadas, sendo uma limitação do estudo.

A maioria dos estudos de avaliação dos efeitos da TRC considerou a presença do distúrbio de condução intraventricular ao ECG de superfície um critério específico de documentação de dissincronia ${ }^{11-14}$. Nesses, a taxa de não-resposta clínica à ressincronização cardíaca oscila entre $20 \%$ e $30 \%{ }^{23}$.

Em nosso estudo, a taxa de não-resposta à TRC foi muito inferior (13,8\%), e isso pode ser explicado pela homogeneidade do padrão de despolarização cardíaca. De fato, o MPC em região apical direita induz de modo uniforme a ativação ápice-base/ VD- VE. Essa modificação, indubitavelmente, é responsável pelo grau máximo de dissincronia ventricular, garantindo assim grande impacto favorável por meio da TRC. Nesse sentido, podemos observar que a duração média do QRS ao ECG de superfície (pré-TRC) foi de $179 \mathrm{~ms}$, valor médio superior ao da casuística de todos os ensaios clínicos clássicos: 174 ms no MUSTIC ${ }^{11} ; 167$ ms no MIRACLE ${ }^{12} ; 159$ ms no COMPANION ${ }^{13}$ e 160 ms nos CARE-HF ${ }^{14}$. Ressalta-se que, embora esse não seja o método padrão de referência para detecção de dissincronia ventricular, existe correlação direta dessa e a duração do QRS'1.

Em nosso estudo, a redução da duração do QRS estimulado (21,4 ms) pós-TRC não se correlacionou com a taxa de resposta clínica ou com a melhora dos parâmetros ecocardiográficos. Esse dado é semelhante ao encontrado em diversos estudos que avaliaram a redução da duração do QRS estimulado após a TRC ${ }^{24}$. No entanto, resultado divergente pode ser encontrado em trabalho isolado de Horwich e cols. ${ }^{1}$, no qual houve correlação entre a redução da duração do QRS pós TRC (23,08 ms) e melhora em parâmetros ecocardiográficos.

No presente estudo, a ocorrência de remodelamento reverso, expresso pelas modificações ecocardiográficas, foi similar a outros estudos que incluíram tanto pacientes com BRE espontâneo como induzido por MPC ${ }^{1,4,12,14,15}$. O achado mais relevante foi a melhora da FEVE em $18 \%(P=0,013)$; correspondendo a um incremento médio absoluto de 5\% nesse parâmetro. Nos estudos MIRACLE ${ }^{11}$ e CARE-HF ${ }^{14}$, houve, respectivamente, aumento médio absoluto na FEVE de 4,6\% e 6,9\%. Já Baker e cols. ${ }^{3}$ e Horwich e cols. ${ }^{1}$ demonstraram aumento médio de $6 \%-7 \%$ avaliando portadores de MPC submetidos à TRC.

A redução dos diâmetros intracavitários, observada no presente estudo, (3,5 $\mathrm{mm}$ do DDVE e 4,3 $\mathrm{mm}$ do DSVE), apesar de não demonstrar significância estatística, corrobora os efeitos da TRC sobre o remodelamento reverso. Nesse sentido, considerando uma maior casuística, em sub-análise de parâmetros ecocardiográficos do estudo MIRACLE ${ }^{25}$, demonstrou-se redução média significativa de 3 e $6 \mathrm{~mm}$ no DDVE e DSVE após seis meses da TRC.

Estudos clínicos em pacientes com IC e disfunção ventricular e pós-infarto do miocárdio demonstraram que o tamanho e o volume do VE têm valor prognóstico para a ocorrência de eventos cardiovasculares adversos, incluindo piora da CF-IC e morte súbita cardíaca. Nesses ensaios, a atenuação da dilatação ou o remodelamento reverso do VE parece reduzir a taxa desses eventos adversos durante o seguimento clínico ${ }^{23}$. Portanto, o remodelamento reverso promovido pela TRC pode ser um dos mecanismos associados aos benefícios dessa terapia.

São necessários, entretanto, novos estudos prospectivos em maior escala e com grupo controle para serem somados aos resultados desse estudo. Além disso, com a evolução contínua da tecnologia, importantes ferramentas diagnósticas, como a ecocardiografia tecidual e a ressonância nuclear magnética cardíaca, deverão aumentar ainda mais o rigor de seleção de pacientes, e assim, melhorar a custo-efetividade da TRC.

\section{Conclusão}

Pacientes com cardiomiopatia dilatada, IC grave refratária ao tratamento clínico e BRE induzido pelo MPC (estimulação apical de VD) se beneficiam da terapia de ressincronização cardíaca. A taxa de responsividade clínica é elevada $(86,2 \%)$ e ocorre melhora significativa da FEVE (18\%) ao ecocardiograma. Desse modo, apesar de serem necessários estudos em maior escala, nossos resultados indicam que, nessa população específica, a ressincronização cardíaca é uma terapia exeqüível, com alta efetividade e não associada 
Silva e cols.

Ressincronização Cardíaca em Portadores de Marcapasso Convencional

\section{Artigo Original}

a complicações cirúrgicas.

\section{Potencial Conflito de Interesses}

Auxílio financeiro para participação em congressos; Apoio financeiro a outros projetos de pesquisa de todas as empresas de marcapasso (Biotronik, ST Jude, Medtronic); honorários para consultoria e palestras da empresa ST Jude.

\section{Referências}

1. Horwich T, Foster E, De Marco T, Tseng Z, Saxon L. Effects of resynchronization therapy on cardiac function in pacemaker patients "upgraded" to biventricular devices. J Cardiovasc Electrophysiol. 2004;15 (11): 1284-9.

2. Baldasseroni S, Opasich C, Gorini M, Lucci D, Marchionni N, Marin $M$, et al, Italian Network on Congestive Heart Failure Investigators: Left bundle-branch block is associated with incresead 1 -year sudden and total mortality rate in 5517 outpatients with congestive heart failure: a report from the Italian Network on Congestive Heart Failure. Am Heart J. 2002 143: 398-405.

3. Baker CM, Christopher TJ, Smith P, Langberg J, Delurgio DB, Leon AR Addition of a left ventricular lead to conventional pacing systems in patients with congestive heart failure: feasibility, safety, and early results in 60 consecutive patients. Pacing Clin Electrophysiol. 2002; 25: 1166-71.

4. Leon AR, Greenberg JM, Kanuru N, Baker CM, Mera FV, Smith AL, et al. Cardiac resynchronization in patients with congestive heart failure and chronic atrial fibrillation. J Am Coll Cardiol. 2002; 39 (8): 1258-63.

5. Vassalo AJ, Cassidy DM, Miller JM, Buxton AE, Marchlinski EF, Josephson EM. Left ventricular endocardial activation during right ventricular pacing. effect of underlying heart disease. J Am Coll Cardiol. 1986; 7 (6): 1228-33.

6. Vernooy K, Verbeek XAAM, Peschar M, Prinzen FW. Relation between abnormal impulse conduction and heart failure. J Interv Cardiol. 2003; 16 (6): 557-62.

7. Wilkoff BL, CookJR, Epstein AE, Greeme HL, Hallstrom AP, Hsia H, et al. Dualchamber pacing or ventricular back-up pacing in pacients with as implantable defibrillator: the Dual Chamber and VVI Implantable Defibrillator (DAVID) Trial. JAMA. 2002; 288: 3115-23.

8. Moss AZ, Zareba W, Hall WJ, Klein H, Wilber DJ, Cannom DS, et al. Prophylatic implatation of a defibrillator in a patients with myocardial infarction and reduced ejection fraction. N Engl J Med. 2002; 346: 877-83.

9. Lamas GA, Lee KL, Sweney MO, Silverman R, Leon A, Yee R, et al. Ventricula pacing or dual-chamber pacing for sinus-node dysfunction. N Engl J Med. 2002; 346 (24): 1854-62.

10. Doshi R, Daoud E, Fellows C, Turk K, Duran A, Hamdan M, et al. Left ventricular-based cardiac stimulation Post AV Nodal Ablation Evaluation (The PAVE Study). J Cardiovasc Electrophysiol. 2005; 16: 1160-5.

11. Cazeau S, Leclercq C, Lavergne T, Walker S, Varma C, Linde C, et al. Effects of multisite biventricular pacing in patients with heart failure and intraventricular conduction delay. N Engl J Med. 2001; 344: 873-80.

12. Abraham WT, Fisher WG, Smith AL, Delurgio DB, Leon AR, Loh E, et al. Cardiac resynchronization in chronic heart failure. N Engl J Med. 2002; 346: 1845-53.

\section{Fontes de Financiamento}

O presente estudo não teve fontes de financiamento externas.

\section{Vinculação Acadêmica}

Não há vinculação deste estudo a programas de pósgraduação.

13. Bristow M, Saxon LA, Boehmer J, Krueger S, Kass DA, De Marco T, et al. for the Comparison of Medical Therapy, Pacing, and Defibrillation Heart Failure (COMPANION) Investigators: cardiac resynchronization therapy with or without an implatable defibrillator in advanced chronic heart failure. N Eng I Med. 2004; 350: 2140-50.

14. Cleland JGF, Daubert JC, Erdmann E, Freemantle N, Gras D, Kappenberger $\mathrm{L}$, et al. for the Cardiac Resynchronization - Heart Failure (CARE-HF) Study Investigators. The effect of cardiac resynchronization on morbidity and mortality in heart failure. N Engl J Med. 2005; 352 (15): 1539-49.

15. Höijer Cl, Meurling C, Brandt J. Upgrade to biventricular pacing in patient with conventional pacemakers and heart failure: a double-blind, randomized crossover study. Europace. 2006; 8: 51-5.

16. Dilaveris P, Pantazis A, Giannopoulos G, Synetos A, Gialafos J, Stefanadis C. Upgrade to biventricular pacing in patients with pacing-induced heart failure: can resyncronization do the trick? Europace. 2006; 8: 352-7.

17. Castro PF, Mc-Nab P, Quintana JC, Bittner A, Greig D, Vergara J, et al. Effects of carvedilol upon intra and interventricular synchrony in patients with chronic heart failure. Am J Cardiol. 2005; 96: 267-9

18. Reuter S, Garrigue S, Barold SS, Jais P, Hocini M, Haissaguerre M, et al. Comparison of characteristics in responders versus nonresponders with biventricular pacing for drug-resistant congestive heart failure. Am J Cardiol. 2002 : $89 \cdot 346-50$

19. Molhoek SG, Bax JJ, Erven L, Bootsma M, Boersma E, Steendijk P, et al. Comparison of benefits from cardiac resynchronization therapy in patients with ischemic cardiomyiopathy versus idiopathic dilated cardiomyiopathy. Am J Cardiol. 2004; 93: 860-3.

20. Cleland JGF, Marang R. Trial design for heart failure studies. Eur Heart J. 1999; 20: $1600-1$.

21. Rostagno C, Galantini G, Comeglio M, Boddi V, Olivo G, Gastoni Neri Serneri G. Comparison of different methods of functional evaluation in patients with chronic heart failure. Eur J Heart Fail. 2000; 2: 273-80.

22. Bennett JA, Riegel B, Bittner V, Nichols J. Validity and reability of the NYHA classes for measuring research outcomes in patients with cardiac disease. Heart Lung. 2002; 31: 262-70.

23. Kass DA. Ventricular resychronization: pathophysiology and identification of responders. Rev Cardiovasc Med. 2003; 4 (Suppl 2): S3-S13.

24. Kashani A, Barold SS. Significance of QRS complex duration in patients with heart failure. J Am Coll Cardiol. 2005; 46: 2183-92.

25. Sutton MGSJ, Plappert T, Abraham WT, Smith AL, Delurgio DB, Leon AR, et al. Effect of cardiac resynchronization therapy on left ventricular size and functional in chronic heart failure. Circulation. 2003; 107: 1985-90. 